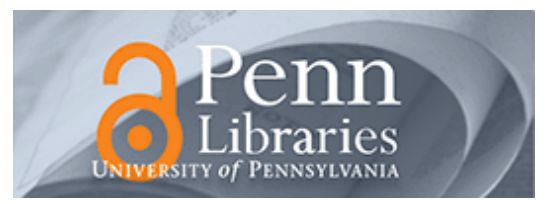

University of Pennsylvania

ScholarlyCommons

May 1994

\title{
Further progress in robot juggling: solvable mirror laws
}

\author{
Alfred A. Rizzi \\ University of Michigan \\ Daniel E. Koditschek \\ University of Pennsylvania, kod@seas.upenn.edu
}

Follow this and additional works at: https://repository.upenn.edu/ese_papers

\section{Recommended Citation}

Alfred A. Rizzi and Daniel E. Koditschek, "Further progress in robot juggling: solvable mirror laws", . May 1994.

Copyright 1994 IEEE. Reprinted from Proceedings of the IEEE International Conference on Robotics and Automation, Volume 4, 1994, pages 2935-2940.

This material is posted here with permission of the IEEE. Such permission of the IEEE does not in any way imply IEEE endorsement of any of the University of Pennsylvania's products or services. Internal or personal use of this material is permitted. However, permission to reprint/republish this material for advertising or promotional purposes or for creating new collective works for resale or redistribution must be obtained from the IEEE by writing to pubs-permissions@ieee.org. By choosing to view this document, you agree to all provisions of the copyright laws protecting it.

NOTE: At the time of publication, author Daniel Koditschek was affiliated with the University of Michigan. Currently, he is a faculty member in the Department of Electrical and Systems Engineering at the University of Pennsylvania.

This paper is posted at ScholarlyCommons. https://repository.upenn.edu/ese_papers/380

For more information, please contact repository@pobox.upenn.edu. 


\title{
Further progress in robot juggling: solvable mirror laws
}

\author{
Abstract \\ In previous papers we have reported successful laboratory implementations of a family of juggling \\ algorithms. In all but the one degree of freedom case, these empirically successful algorithms have so far \\ resisted our analytical efforts to explain why they work. This is in large measure a consequence of our \\ inability to write down using elementary functions an expression for the closed loop dynamics they \\ induce. We discuss in this paper a modified juggling algorithm whose resulting closed loop dynamics can \\ be written down directly. We offer data establishing the empirical success of the new algorithm. \\ Theoretical analysis of the closed loop dynamics is presently in progress.

\section{Comments} \\ Copyright 1994 IEEE. Reprinted from Proceedings of the IEEE International Conference on Robotics and \\ Automation, Volume 4, 1994, pages 2935-2940. \\ This material is posted here with permission of the IEEE. Such permission of the IEEE does not in any way \\ imply IEEE endorsement of any of the University of Pennsylvania's products or services. Internal or \\ personal use of this material is permitted. However, permission to reprint/republish this material for \\ advertising or promotional purposes or for creating new collective works for resale or redistribution must \\ be obtained from the IEEE by writing to pubs-permissions@ieee.org. By choosing to view this document, \\ you agree to all provisions of the copyright laws protecting it. \\ NOTE: At the time of publication, author Daniel Koditschek was affiliated with the University of Michigan. \\ Currently, he is a faculty member in the Department of Electrical and Systems Engineering at the \\ University of Pennsylvania.
}




\title{
Further Progress in Robot Juggling: Solvable Mirror Laws
}

\author{
Alfred A. Rizzi *and Daniel E. Koditschek ${ }^{\dagger}$ \\ Artificial Intelligence Laboratory \\ University of Michigan \\ Department of Electrical Engineering and Computer Science
}

\begin{abstract}
In previous papers we have reported successful laboratory implementations of a family of juggling algorithms. In all but the one degree of freedom case, these empirically successful algorithms have so far resisted our analytical efforts to explain why they work. This is in large measure a consequence of our inability to write down using elementary functions an expression for the closed loop dynamics they induce. We discuss in this paper a modified juggling algorithm whose resulting closed loop dynamics can be written down directly. We offer data establishing the empirical success of the new algorithm. Theoretical analysis of the closed loop dynamics is presently in progress.
\end{abstract}

\section{Introduction}

For the last five years we have been building and theorizing about machines that bat one or two balls into stable periodic vertical orbits. The study of such tasks has design implications for locomotion [7], for dexterous manipulation [2], and for sensory attention [10] problems in robotics. Our achievement of the spatial two juggle, reported in last year's ICRA conference [9], brings this effort to an interesting juncture. Our ability to build robust laboratory demonstrations of various dexterous manipulation tasks has now considerably outstripped our ability to analyze the causes of their success. In this paper we take a step back from the building and behavioral recording to report on some recent analytical work in progress.

\footnotetext{
* Supported in part by IBM through a Manufacturing Graduate Fellowship and in part by the National Science Foundation under grant IRI-9123266.

'Supported in part by the National Science Foundation under grant IRI-9123266, and in part by the Special Semester on Spatial and Temporal Dynamics of the Université de Montréal Centre de Recherches Mathématiques.
}

Most generally, what we seek (and do not presently have) is a systems theory for coupled oscillators. ${ }^{1}$ By this we mean a qualitative theory that permits the interconnection of oscillators in such a manner that the limit set of the coupled system is the cross product of the individual limit sets and, in the event that both are limit cycles, affords a tuning procedure for their relative phase. Despite more than four decades of interest in such a nonlinear theory [15], this understanding does not yet appear to be at hand. The present paper reviews our application of a strong regulation theory for one degree of freedom oscillators and documents our efforts to analyze some specific instances of coupling between two and three degree of freedom oscillators.

We are interested in the motion of partially actuated mechanisms that interact in an intermittent and dynamical fashion with their environment. When considering legged locomotion or juggling, the environment is modeled as gravity pulling masses toward ground: individual cases vary according to the contact model and actuation strategy. The exchange of kinetic and potential energy guarantees oscillatory behavior in physically viable hopping and juggling machines. However, the designer must devise a strategy for imparting energy via the available actuators in such a fashion that the desired motion is regulated. The presumption of hopping and juggling machine designers has been that a stable limit cycle in the vertical degree of freedom affords the possibility of "entraining" other purposive rhythms in the transverse plane $[2,8]$. Empirical success suggests the validity of this presumption. But it is crucial to put these ideas on a sound theoretical footing as well.

The paper is organized as follows. The next sec-

\footnotetext{
${ }^{1}$ Throughout this paper the term oscillator denotes a nonlinear dynamical system with a globally attracting compact forward limit set. For example, point attractors are trivial oscillators. The term tunable denotes oscillators that persist (despite likely bifurcations in the limit set topology) over large volumes in the parameter space that defines the dynamics.
} 
tion offers a tutorial account of a one degree of freedom version of our juggling algorithms: its theoretical properties are well understood. Section 3 shows how these algorithms generalize to higher degrees of freedom and afford extremely robust laboratory implementations but resist analytical understanding: they induce closed loop dynamics that cannot be written in terms of elementary functions. Section 4 presents successful empirical results for a modified family of algorithms whose induced closed loop dynamics can be written down directly. We are hopeful that the availability of the closed loop expression will permit a complete stability analysis elucidating the manner in which horizontal convergence is yoked to the vertical "heartbeat" in our machines.

\section{A Regulation Theory for One Degree of Freedom Oscillators}

This section reviews the case of an analytically straightforward one degree of freedom batting algorithm whose feedback structure will be generalized to less analytically tractable (but empirically successful) higher degree of freedom analogues in the sequel. We will merely allude to the parallels with hopping machine studies [7]. We first introduce the notion of a return map and then sketch the theory of S-unimodal return maps developed two decades ago within the dynamical systems community. The "gedanken juggler" [3] is used to exemplify these theoretical ideas.

\subsection{Return Maps}

A standard analytical tool for investigating the stability of limit cycles is the return map [5]. Let the dynamical system $\dot{x}=f(x)$ generate a flow $f^{t}(x)$ (an integral curve through the initial condition). A codimensionone manifold, $\Sigma \subset \mathcal{X}$ results from the zero set of a smooth scalar valued map, $\sigma: \mathcal{X} \rightarrow I R$, that is, $\Sigma=\sigma^{-1}[0]$, as long as $\sigma \mid \Sigma$ has a full rank jacobian. Suppose there is such a $\Sigma$ through which the flow passes repeatedly - that is, after some finite interval of time, $[0, T]$, solutions starting in $\Sigma$ will return,

$$
\Sigma \subset \bigcup_{t<T} f^{t}(\Sigma)
$$

Every $x \in \Sigma$ has a time of first return, $\tau(x)$. The function $\Phi(x) \triangleq f^{\tau(x)}(x)$ is called the return map.

The function $\Phi$ generally must be derived first by solving the differential equation to get $f^{t}$ and then by solving for $\mathrm{t}$ the equation

$$
\sigma \circ f^{t}(x)=0
$$

to get $\tau$.

\subsubsection{The Mirror Law for a Gedanken Robot}

As an example, consider the gedanken juggler - a ball, $b$ and robot, $r$, both confined to a vertical line in space [1]. Their dynamics are described in the phase variables,

$$
w \triangleq\left[\begin{array}{l}
b \\
\dot{b}
\end{array}\right] \text { and } v \triangleq\left[\begin{array}{l}
r \\
\dot{r}
\end{array}\right],
$$

as

$$
\begin{aligned}
\dot{x} & =F(x):=A x+b u \\
& A=\left[\begin{array}{ll}
0 & 1 \\
0 & 0
\end{array}\right] ; \quad b=\left[\begin{array}{l}
0 \\
1
\end{array}\right]
\end{aligned}
$$

where $u=-\gamma$ is the gravitational constant when $x=$ $w$ and and $u=\zeta$ is the force applied to the robot's shaft when $x=v$.

The body's integral curves are given by

$$
F^{t}(w):=\left[\begin{array}{ll}
1 & t \\
0 & 1
\end{array}\right] w-\gamma t\left[\begin{array}{c}
t / 2 \\
1
\end{array}\right]
$$

We model collisions between the robot and the ball using the simple (but standard [14]) coefficient of restitution law, leading to the collision map

$$
\begin{aligned}
C_{v}(w) & :=R w+(I-R) v \\
& R=\left[\begin{array}{cc}
1 & 0 \\
0 & -\alpha
\end{array}\right] ; 0 \leq \alpha \leq 1
\end{aligned}
$$

relating the body state after a collision to the body and robot states before collision.

The juggling algorithm for this system, the mirror law introduced by Bühler [1], describes the motion of the robot as a function of $b$, and takes the form

$$
r=\mu(b, \dot{b}):=k(w) \cdot b
$$

where $k(w)=\left(\kappa_{0}+\kappa_{1}\left(\eta^{*}-\eta\right)\right)$, with $\eta=\frac{1}{2} \dot{b}^{2}+\gamma b$ the scaled total energy of the ball, and $\eta^{*}$ the desired total energy for the specified juggling task.

\subsubsection{Computation of the Return Map}

Assuming that some force law, $\zeta$, is applied to the robot in (2) causing $r$ to track $\mu(w)$ by the time an impact occurs, we are guaranteed that the ball will fall until

$$
b=\mu(b, \dot{b}) \text {. }
$$

Imposing the (physically necessary) assumption that

$$
k(w)<1,
$$


implies that collisions only take place when

$$
\sigma(b, \dot{b}):=b=0
$$

so we take $\Sigma$ to be the abscissa of the ball's phase plane. The body collides, then flies,

$$
\tilde{F}^{t}(w):=F^{t} \circ C_{\dot{r}}(w)
$$

until the next impact. Thus in order to compute the return map, it remains to determine the robot's velocity at impact, $\dot{r}$, and the time of flight, $t$, both as functions of $w$.

Since we assume no energy loss in $\operatorname{flight}(k(w)=0)$, the robot's velocity at impact is

$$
\dot{r}=k(w) \cdot \dot{b}=: \delta(w)
$$

Since impacts always occur on $\Sigma$, we obtain a simple solution for time of flight ${ }^{2}$

$$
\tau(w):=\left[C_{\delta(w)}(w)\right]_{2} 2 / \gamma
$$

The return map can now be written explicitly as

$$
\begin{aligned}
\Gamma(w) & :=F^{\tau(w)} \circ C_{\delta(w)}(w) \\
& =\left[\begin{array}{c}
0 \\
\dot{b}\left[1-\beta\left(\dot{b}^{2}-\dot{b}^{* 2}\right)\right]
\end{array}\right]
\end{aligned}
$$

where $\beta=\frac{1+\alpha}{2} \kappa_{1}$.

For reasons to become clear below we choose to rewrite this return map in "apex" coordinates (i.e. to measure the height of the ball at apex rather than the velocity pre-impact). In these coordinates we have

$$
\bar{\Gamma}(\bar{w}):=\left[\begin{array}{c}
(\alpha+k(\bar{w})(1+\alpha))^{2} \bar{b} \\
0
\end{array}\right]
$$

\subsection{Unimodal Return Maps}

We have developed simplified models of a variety of dynamically dexterous machines that permit the exact derivation of $\Gamma$, as above. For example, simple hopper models result from considering the purely vertical mode of Raibert's machine [8] - an elastic mass that falls to the ground, exchanges its kinetic energy for some potential energy stored in the elastic spring, then rises and eventually leaves the ground as the spring, further restored by energy contributions from an actuator, works against gravity. The various stages of flight, touch down, stance, and liftoff are modeled by

\footnotetext{
${ }^{2}$ Here and in the sequel we will use []$_{i}$ to represent the $i^{\text {th }}$ component of a vector, or vector valued function.
}

distinct mechanical systems whose initial and final conditions are pieced together to obtain a discrete model of the return dynamics [7], similarly as above.

In developing these one degree of freedom hopper and juggler models, we have encountered an unusually strong stability mechanism. In the cases considered carefully to date, the return map, $\Gamma$ is a unimodal function: there is a single critical point and the function is monotone up or down on respective sides. Moreover, the Schwartzian derivative [5] of each is negative. Singer showed that in this case there can be at most one attracting periodic orbit [13]. Guckeheimer showed the domain of attraction of such a periodic orbit is almost all of the state space [6]. We take this as a model of regulation theory.

It is easy to see in (8) that $\Gamma$ has a single critical point over the regime of physical interest (the real interval bounded by the two zeroes of the cubic function). Moreover, since the second component of $\Gamma$ has all real roots, we are assured of a negative Schwartzian [4]. The Singer Guckenheimer Threory now applies immediately.

\section{Missing: A Systems The- ory for Intermittent Dynamic Tasks}

In this section we present two and three degree of freedom generalizations of the gedanken juggler introduced above. We will point out two major obstacles to extending the regulation theory described:

- the time of flight (1) must be explicitly solved to get $\Gamma$

- The Singer-Guckenheimer theory of S-unimodal maps applies only to one-dimensional maps

The first of these obstacles is mitigated in the next section by the substitution of a new mirror law (15). Addressing the second remains the principle focus of our theoretical work in progress on this problem.

\subsection{A General Family of Robot Con- trol Laws for Intermittent Dynam- ical Tasks}

Our research has concerned the development and implementation of a class of control algorithms suitable for performing "robot juggling". This has lead to the emergence of an increasingly coherent set of rules for generalizing these algorithms to machines with more degrees of freedom and different kinematic structure. 
What follows is an application of these rules in the specific context of a planar and a spatial juggling machine.

\subsubsection{The Planar Juggler}

Consider the planar juggling task, which was the central topic of [3]. In this example the ball is free to travel on an inclined two dimensional plane. Thus the particular environmental dynamics (3) are given by

$$
\ddot{b}=a_{p}
$$

where $b \in I R^{2}$, and $a_{p}=(0,-\gamma)^{T}$ is the acceleration due to gravity. The "actuator" used to impart control over this ball is a one degree of freedom robot with a long bar attached to it. In this case the kinematics are given by

$$
g^{-1}(b) \triangleq\left[\begin{array}{c}
\arctan \left(b_{z} / b_{x}\right) \\
\sqrt{b_{x}^{2}+b_{z}^{2}}
\end{array}\right]
$$

Following a strategy similar to that used for the gedanken juggler, the algorithm for this system describes a reference trajectory (angle as a function of time) for the robot to track as a function of the ball's state $^{3}$ :

$$
r=m(w):=k(w) \cdot\left[g^{-1}(b)\right]_{1}+l(w)
$$

where $k(w)$ is defined as in (5), and $l$ is an affine linear map implementing proportional and derivative spatial regulation terms:

$$
l(w):=\kappa_{10}\left(b_{x}-b_{x}^{*}\right)+\kappa_{11} \dot{b}_{x} .
$$

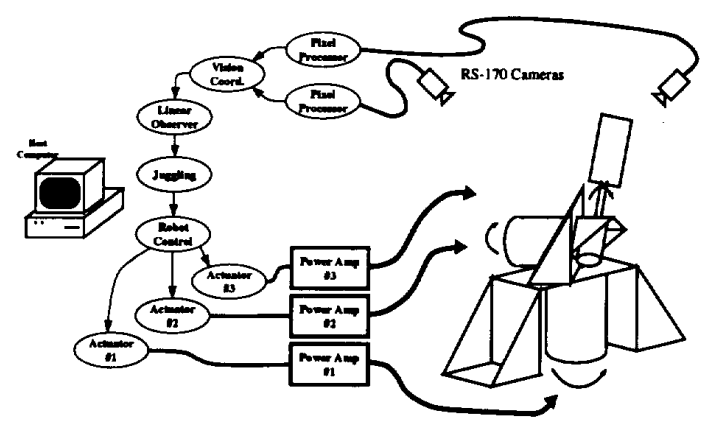

Figure 1: The spatial juggling system.

\footnotetext{
${ }^{3}\left[g^{-1}\right]_{i}$ is used here, as in (7), to represent the $i^{\text {th }}$ component of the inverse kinematic image of the ball.
}

\subsubsection{The Spatial Juggler}

Next consider the spatial juggling task as implemented on the system shown in Figure $1[12,9,11]$. In this case we consider a three degree of freedom falling ball, whose dynamics are identical to (3) save that $b \in I R^{3}$ and $a_{p}=(0,0,-\gamma)^{T}$. This ball is "actuated" by a three degree of freedom robot. As with the planar juggler, the juggling algorithm is constructed by first taking the inverse kinematic image of the ball, then "mirroring" it with the robot. Briefly, the resulting algorithm takes the form

$$
m(w):=\left[\begin{array}{c}
{\left[g^{-1}\right]_{1}(b)} \\
-\frac{x}{2}-k\left(\left[g^{-1}\right]_{2}(b)+\frac{\pi}{2}\right)+-l_{1}(b, \dot{b}) \\
l_{2}(b, \dot{b})
\end{array}\right],
$$

where as in the planar case $l_{1}$, and $l_{2}$ are linear affine transformations implementing proportional-derivative spatial regulation terms in the radial and lateral directions.

\subsection{Unsolvable Return Map}

Our interest in generalizing these algorithms is contingent upon their correctness. In each case the coupled robot and environment gives rise to a discrete dynamical systems sampled by the event of the ball impacting the robot. The stability of this system comprises the feature whose correctness is to be demonstrated.

Even for the "simple" planer machine attempting the analysis of Section 2.1 is not fruitful here. The primary difficulty arises from the unsolvability of time of flight: computation of $\tau$ from (1) which requires solution of the transcendental equation

$$
\arctan \left(\frac{b_{x}}{b_{x}}\right)=k(w) \cdot \arctan \left(\frac{b_{x}}{b_{x}}\right)+l\left(b_{x}, \dot{b}_{x}\right)
$$

when $b_{x}, b_{z}$, and $b_{x}$ are replaced by their appropriate time functions. No closed form solution exists for this equation, and thus derivation of an explicit return map is not possible. This has impeded a global closed loop analysis.

Not surprisingly, similar difficulties arise with the spatial juggling system, although in this case the resulting transcendental equation is significantly more complicated due to the more complicated kinematics. 


\section{A Modified Mirror law}

\subsection{Solvable Time of Flight}

One variant of the planar mirror law which will afford a solvable time of flight is

$$
\begin{aligned}
r=m(w):= & k(w) \cdot\left[g^{-1}\right]_{1}(b)+ \\
& (1-k(w)) \cdot\left[g^{-1}\right]_{1}\left(l \circ F^{t_{a}}(w)\right)
\end{aligned}
$$

where $k(w)$ is defined as in (5), but now

$$
l(w) \triangleq\left[\kappa_{10}\left(b_{x}-\overline{b_{x}}\right)+\kappa_{11} \dot{b}_{x}, b_{x}\right],
$$

and $F^{t_{a}}$ is the newtonian flight map which returns a ball $w$ to its apex $\left(t_{a}(w):=\frac{\dot{b}_{x}}{\gamma}\right)^{4}$. This "new" mirror law eliminates the transcendental structure of (14) by passing the spatial regulation terms through the inverse kinematics of the machine, and simplifies the computation by writing the proportional derivative terms in the ball's apex frame. Thus the time of flight computation (1) is reduced to solving

$$
b_{z}=\frac{\left[l_{1}(\bar{w})\right.}{1-k}
$$

for $t$ after substituting the appropriate time functions for $b_{z}{ }^{5}$, where $\bar{w}$ is the apex location from which the ball fell.

Making similar use of the inverse kinematics of the robot we can construct a solvable time of flight mirror law for the spatial juggler as well. In this case the "new" mirror law will take a form related to (13) reminiscent of that relating (15) to (12).

\subsection{Closed Loop Return Map}

Given the closed form solution for (1) (which now taked the specific form of (16)), we are in a position to exhibit the closed loop dynamics induced by (15). Since $\Sigma$ has co-dimension one, we expect a three dimensional discrete system.

For reasons explained below, we choose to work in "apex" coordinates. More formally, using over-bars to denote states in the apex coordinates we have

$$
\bar{w}_{k}=h(w):=F^{t_{a}}(w)
$$

where $t_{a}(w) \triangleq \frac{\dot{b}_{x}}{\gamma}$ is the time of flight to the apex of flight.

\footnotetext{
"Note that $l(w)$ is now a vector valued function mapping the phase space of the ball onto the ball's configuration space.

${ }^{5}$ For a ball falling under the influence of gravity this is a quadratic equation.
}

The return map in these coordinates is now ${ }^{6}$

$$
\bar{w}_{k+1}=\Phi\left(\bar{w}_{k}\right):=F^{t_{\star}} \circ C_{\delta\left(\bar{w}_{k}\right.} \circ F^{\tau_{k}}\left(\bar{w}_{k}\right) .
$$

Explicitly, $\Phi$ may be simplified to the form.

$$
\Phi(w):=\left[\begin{array}{c}
\bar{b}_{x}+\dot{\bar{b}}_{x}\left(\tau_{k}+t_{a}\right)+(1+\alpha)(k+1) \dot{\theta}_{b} t_{a} l\left(\bar{b}_{x}, \dot{\bar{b}}_{x}\right) \\
\Gamma\left(\bar{b}_{x}\right)+l\left(\bar{b}_{x}, \overline{\bar{b}}_{x}\right) d\left(\bar{w}_{k}\right) \\
\dot{\bar{b}}_{x}+(1+\alpha)(k+1) \dot{\theta}_{b} l\left(\bar{b}_{x}, \dot{b}_{x}\right) \\
0
\end{array}\right] .
$$

Here $d(w)$ represents the coupling of the horizontal system back into the vertical, and $\Gamma$ is the return map of (8). In particular

$$
d(\bar{w})=1-\frac{\xi_{0}^{2}}{\gamma^{2}}+2 \xi_{0} \xi_{1} \tau_{k} l(\bar{w})+\xi_{1}^{2} l^{3}(\bar{w})
$$

where

$$
\xi_{0}:=(\alpha+k \cdot(1+\alpha)) \gamma
$$

and

$$
\xi_{1}:=(1+\alpha)(1+k) \frac{\dot{\rho}_{b}}{\|b\|} .
$$

Finally $\dot{\theta}_{b}$ and $\dot{\rho}_{b}$ denote to the time derivatives of $\left[g^{-1}\right]_{1}(b)$ and $\left[g^{-1}\right]_{2}(b)$ respectively.

We find this form particularly attractive since it expresses the behavior of the overall planer system in terms of the better understood "line-juggler" of Section 2 and a nearly linear dynamical system representing the "horizontal" evolution. This structure is revealed by rewriting (20) as

$$
\begin{aligned}
& \bar{b}_{x}(k+1)=\Gamma\left(\bar{b}_{x}\right)+l\left(\bar{b}_{x}, \dot{\bar{b}}_{x}\right) \cdot d\left(\bar{w}_{k}\right) \\
& {\left[\begin{array}{l}
\bar{b}_{x} \\
\dot{b}_{x}
\end{array}\right](k+1)=\left[\begin{array}{cc}
1 & \left(\tau_{k}+t_{a}\right) \\
0 & 1
\end{array}\right]\left[\begin{array}{c}
\bar{b}_{x} \\
\bar{b}_{x}
\end{array}\right](k)+\xi_{2}\left[\begin{array}{c}
t_{a} \\
1
\end{array}\right] l,}
\end{aligned}
$$

where $\xi_{2}:=(1+\alpha)(k+1) \dot{\theta}_{b}$. In this form the effect of $l$ on the horizontal subsystem $\left(b_{x}\right)$ can clearly be seen as a (stabilizing) input, while it's coupling to the vertical system is scaled by $d$, which will vanish with $\bar{b}_{x}$ and $\dot{\bar{b}}_{x}$. The effect of the vertical subsystem on the horizontal behavior is slightly less clear, as the primary coupling is through the time of flight ( $\tau_{k}$ and $t_{a}$ ).

\subsection{Experimental Results}

We would not wish to waste our efforts by attempting to prove correctness for a class of algorithms which do not function! To validate these new ideas in the absence of theoretical stability guarantees, we have implemented a slightly modified version of (13) on the

\footnotetext{
${ }^{6}$ Although $\Phi$ looks more algebraically complicated than $\Gamma$ from (8), the apex coordinates force $\bar{\Sigma}$ to be a linear subspace, $\left\{\dot{b}_{z}=0\right\}$, which $\Gamma$ leaves invariant. In contrast, the equivalent three dimensional invariant manifold, $\Sigma$, left invariant by return map taken at impact is a rather complicated algebraic surface.
} 
spatial juggling machine. In practice the performance of the "modified" mirror law is nearly indistinguishable from the algorithms which did not admit explicit computation of time-of-flight.

\section{Conclusion}

Although it seems like a modest step, deriving a closed form expression for the return map generated by our juggling robots represents a significant advance for us. We are finally able to display the dynamics of the two degree of freedom system (19) in a fashion that shows the component one degree of freedom dynamics (a unimodal map for the vertical subsystem; a PD compensated double integrator for the horizontal subsystem) and the manner in which they are coupled together.

There still remains the important step of demonstrating that the coupling of these two subsystems does not destroy the stability of the two in isolation. We expect a proof of this to take the following basic form:

1. Demonstrate an upper and lower bound on $\tau_{k}$ and $t_{a}$.

2. Show robust stability of the horizontal subsystem for $t_{k}$ and $t_{a}$ in this interval.

3 . Conclude by exhibiting stability of $\Gamma$ from (8) even in the presence of exponentially decaying disturbances (i.e. from $d(\cdot)$ in (19)) .

Finally, we are hopeful that, when completed, this stability argument will generalize to systems where $\tau$ is available only in the implicit from indicated in (1). This would greatly advance the prospect of for deriving practical design principles from our juggling work.

\section{References}

[1] M. Bühler and D. E. Koditschek. From stable to chaotic juggling. In Proc. IEEE International Conference on Robotics and Automation, pages 1976-1981, Cincinnati, OH, May 1990.

[2] M. Bühler, D. E. Koditschek, and P. J. Kindlmann. Planning and control of a juggling robot. International Journal of Robotics Research, (to appear), 1993.

[3] Martin Bühler. Robotic Tasks with Intermittent Dynamics. PhD thesis, Yale University, New Haven, CT, May 1990.

[4] Robert L. Devaney. Introduction to Chaotic Dynamical Systems. Addison Wesley, Reading, MA, 1987.

[5] J. Guckenheimer and P. Holmes. Nonlinear Oscillations, Dynamical Systems, and Bifurcations of Vector Fields. Springer-Verlag, New York, 1983.
[6] John Guckenheimer. Sensitive dependence to initial conditions for one dimensional maps. Communications in Mathematical Physics, (70):133-160, 1979.

[7] D. E. Koditschek and M. Bühler. Analysis of a simplified hopping robot. International Journal of Robotics Research, 10(6), Dec 1991 .

[8] Marc H. Raibert. Legged Robots That Balance. MIT Press, Cambridge, MA, 1986.

[9] A. A. Rizzi and D. E. Koditschek. Further progress in robot juggling: The spatial two-juggle. In IEEE Int. Conf. Robt. Aut., pages 3:919-924, May 1993.

[10] A. A. Rizzi and D. E. Koditschek. Toward the control of attention in a dynamically dexterous robot. In Koichi Hashimoto, editor, Visual Servoing - Automatic Control of Mechanical Systems with Visual Sensors. World Scientific, 1993 (to appear).

[11] Alfred Rizzi and Daniel E. Koditschek. Preliminary experiments in robot juggling. In Proc. Int. Symp. on Experimental Robotics, Toulouse, France, June 1991. MIT Press.

[12] Alfred A. Rizzi and D. E. Koditschek. Progress in spatial robot juggling. In IEEE Int. Conf. Robt. Aut., pages 775-780, Nice, France, May 1992.

[13] David Singer. Stable orbits and bifurcations of maps of the interval. SIAM J. Applied Mathematics, 35(2):260-267, Sep 1978.

[14] J. L. Synge and B. A. Griffith. Principles of Mechanics. McGraw Hill, London, 1959.

[15] Norbert Wiener. Nonlinear Problems in Random Theory. MIT Press, Cambridge, MA, 1958. 